\title{
Inflammasomes: Intracellular detection of extracellular bacteria
}

Cell Research (2016) 26:859-860. doi:10.1038/cr.2016.67; published online 10 June 2016

The non-canonical inflammasome triggers host cell death and inflammation upon recognition of cytosolic LPS. A recent report in Cell now shows that Outer Membrane Vesicles (OMVs) of extracellular Gram-negative bacteria can deliver LPS into the host cell cytosol.

Lipopolysaccharide (LPS), a component of the outer cell membrane of Gram-negative bacteria, is one of the strongest activators of the immune system. High levels of LPS in the blood as a result of infection can trigger a systemic inflammatory response and, in severe cases, lead to a life-threatening condition known as septic shock. Extracellular LPS is sensed by Toll-like receptor 4 (TLR4), a pattern-recognition receptor that initiates the production of inflammatory cytokines and interferons [1]. LPS is also sensed in the cytosol of host cell by the so-called non-canonical inflammasome [2, 3], which induces pyroptotic cell death and the secretion of pro-inflammatory cytokines like interleukin (IL)-1.

The non-canonical inflammasome pathway involves the binding of LPS to the Caspase Recruitment Domain of caspase-11 in mice or caspase- 4 and -5 in humans [4], which activates these caspases and allows them to initiate downstream signaling events. Since its discovery, caspase-11 has been linked to the recognition of a number of Gram-negative bacteria, including Salmonella typhimurium, Shigella flexneri and Burkholderia thailandensis [5, 6], and shown to drive LPS-induced lethality in a mouse model of septic shock $[2,3]$. It is assumed that caspase- 11 mainly senses intracellular pathogens that escape into the host cell cytosol [7], but unexpectedly, extracellular bacteria were also shown to induce host cell death and cytokine release in a caspase11-dependent manner [6].

Vanaja et al. [8] now report that Outer Membrane Vesicles (OMVs) produced by extracellular bacteria can act as vehicles that deliver LPS into the cytosol of host cells (Figure 1). To determine how extracellular bacteria trigger caspase-11 activation, Vanaja and colleagues focused on enterohemorrhagic Escherichia coli (EHEC), a noninvasive foodborne pathogen that colonizes the intestinal mucosa by adhering to the gut epithelium, causing intestinal inflammation and hemorrhagic colitis. When infecting macrophages with EHEC, they observed that caspase-11 is activated even though they could not detect bacteria in the cytosol of the infected cells. However, foci of LPS could be found in the cytosol of EHECinfected cells, indicating the existence of a yet-unrecognized mechanism that allows the translocation and detection of LPS from extracellular bacteria by caspase- 11 .

How does LPS gain access to the cytosol during EHEC infection? Inhibition of phagocytosis did not alter the amount of LPS detected in the cytosol or caspase-11 activation, and free LPS could not just diffuse across the membrane but required delivery by transfection or electroporation to trigger caspase-11 activation as shown previously [2,3]. Since heat-killed EHEC did not elicit caspase-11 activation, Vanaja and colleagues surmised that a car- rier mechanism unique to live bacteria must be required. Both eukaryotic and prokaryotic cells are known to produce extracellular vesicles, which might allow cell-to-cell communication and the transfer of lipids, proteins and nucleic acids [9]. In Gram-negative bacteria such vesicles derive from the outer cell membrane, hence called OMVs. Previous studies showed that OMVs can stimulate the immune system, for example by delivering peptidoglycan and initiating NOD signaling and NF $\kappa B$ activation [10]. Intriguingly, vesiclelike LPS-containing structures could be detected both extra- and intracellularly during EHEC infection of macrophages, leading the authors to hypothesize that EHEC OMVs might deliver LPS into macrophages. Consistent with this notion, they found that LPS can be detected in the cytosol of cells treated with purified OMVs and that OMV treatment resulted in strong caspase-11 activation.

To investigate how OMVs gain access to the cytosol, Vanaja et al. then tracked their location within OMVtreated macrophages. While internalized OMVs were found in the cytosol at later timepoints, they co-localized with early endosomal compartments immediately after treatment, suggesting entry through endocytosis. Detailed analysis revealed that clathrin-mediated endocytosis (CME) was required for OMV uptake; OMVs co-localized with clathrin and silencing of AP2, a CME adaptor complex, reduced OMV-induced caspase-11 activation. Importantly, OMV appeared to access the cytosol before the early endosomes mature into late endosomes/lysosomes, since blocking 


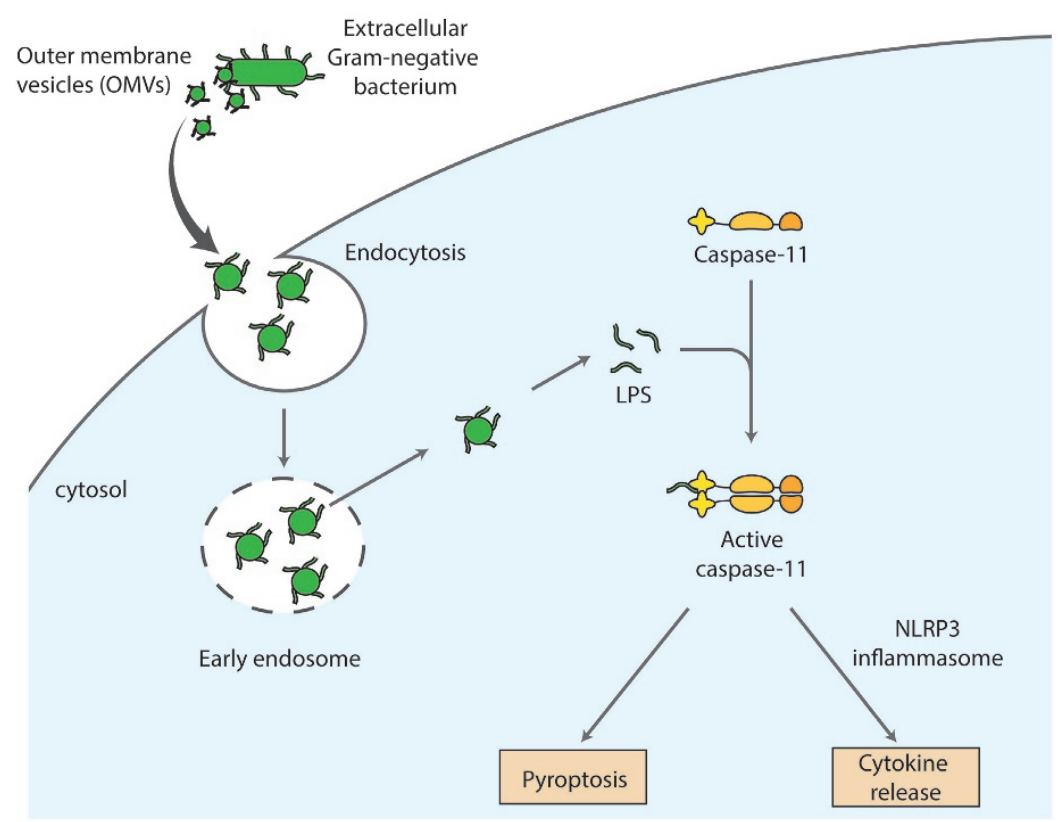

Figure 1 Gram-negative bacteria release LPS-containing Outer Membrane vesicles (OMVs), which can be internalized by host cells through endocytosis. OMVs escape the early endosomes before these mature into late endosomes/lysosomes and translocate to the cytosol. Here, the LPS found in OMVs is sensed by the noncanonical inflammasome, resulting in the activation of caspase-11 in mouse cells or caspase- 4 or caspase- 5 in human cells. These caspases initiate pyroptotic host cell death and activate the non-canonical NLRP3 pathway, which drives caspase1-dependent cytokine maturation.

early-to-late endosome transition did not prevent inflammasome activation.

Overall, these data show that OMVs can translocate LPS into the host cell cytosol, but does this pathway play a relevant role during bacterial infections? To answer this, Vanaja et al. generated hypovesiculating EHEC strains and found that these strains induce reduced caspase-11 activation in macrophages and reduced inflammatory responses after intra-peritoneal injection in mice. Caspase-11 has been shown to mediate host defense against the mucosal pathogen Citrobacter rodentium, which is often used in mouse models of human EHEC infection. Immunogold labeling revealed the presence of OMV-like vesicles in the close proximity of $C$. rodentium and microvilli in the gut, suggesting that Citrobacter also releases OMVs, which might trigger an inflammatory response in the gut epithelium.

The discovery that OMVs can deliver LPS and other PAMPs into the cytosol of host cells is an important step forward in our understanding of both host-pathogen interaction in general and the non-canonical inflammasome in particular. It will be interesting to determine whether this pathway plays a role in the detection of other Gram-negative bacteria and whether caspase-11 activation by intracellular bacteria might also depend on the release of OMVs within cells. How exactly OMVs gain access from early endosomes to the cytosol remains an open question. It is possible that OMV-associated bacterial components such as pore-forming toxin might drive this process, or even that host factors are involved. Furthermore, what happens after LPS-containing OMVs reach the cytosol still remains unclear. Are host proteins involved in liberating LPS from OMVs in order to present it to caspase-11? Or is caspase-11 activated at the OMVs, which as Vanaja et al. speculate contain high local concentrations of LPS and might serve as platforms facilitating caspase-11 oligomerization. LPS is most likely not the only PAMP that is delivered by OMVs. OMVs have been linked to the translocation of peptidoglycan into host cells previously [10], but whether other ligands like DNA or flagellin might be delivered as well, and whether this is sufficient to initiate cytosolic immune responses still need to be shown.

OMVs have recently emerged as a promising vaccine platform, since they derive from the outer cell membrane of bacteria and are highly immunogenic [11]. OMV-based vaccines for N. meningitidis have been approved in some countries, and others targeting a number of pathogens are under development. The finding that OMV-delivered LPS can induce inflammatory responses or even trigger host cell death has thus also important implications for the development of future OMV-based vaccines, and might lead to improvements in their efficacy and safety.

\section{Petr Broz ${ }^{1}$}

${ }^{1}$ Focal Area Infection Biology, Biozentrum, University of Basel, Basel, Switzerland

Correspondence: Petr Broz

E-mail: petr.broz@unibas.ch

\section{References}

1 Poltorak A, He X, Smirnova I, et al. Science 1998; 282:2085-2088.

2 Kayagaki N, Wong MT, Stowe IB, et al. Science 2013; 341:1246-1249.

3 Hagar JA, Powell DA, Aachoui Y, et al. Science $2013 ; \mathbf{3 4 1}: 1250-1253$.

4 Shi J, Zhao Y, Wang Y, et al. Nature 2014; 514:187-192.

5 Broz P, Ruby T, Belhocine K, et al. Nature 2012; 490:288-291.

6 Rathinam VA, Vanaja SK, Waggoner L, et al. Cell 2012; 150:606-619.

7 Aachoui Y, Leaf IA, Hagar JA, et al. Science 2013; 339:975-978.

8 Vanaja SK, Russo AJ, Behl B, et al. Cell 2016; 165:1106-1119.

9 Kulp A, Kuehn MJ. Annu Rev Microbiol 2010; 64:163-184.

10 Kaparakis M, Turnbull L, Carneiro L, et al. Cell Microbiol 2010; 12:372-385.

11 Kuehn MJ, Kesty NC. Genes Dev 2005; 19:2645-2655. 